\title{
An Onyx tunnel: reconstructive transvenous balloon-assisted Onyx embolization for dural arteriovenous fistula of the transverse-sigmoid sinus
}

\author{
${ }^{*}$ Mena G. Kerolus, MD, ${ }^{1}$ Joonho Chung, MD, PhD, ${ }^{1-3}$ Stephen A. Munich, MD, ${ }^{1}$ \\ Yoshikazu Matsuda, MD, ${ }^{1,4}$ Hideo Okada, MD, ${ }^{1,5}$ and Demetrius K. Lopes, MD1

\begin{abstract}
1Department of Neurosurgery, Rush University Medical Center, Chicago, Illinois; '2Department of Neurosurgery, Gangnam Severance Hospital, and ${ }^{3}$ Severance Institute for Vascular and Metabolic Research, Yonsei University College of Medicine, Seoul, Republic of Korea; ${ }^{4}$ Department of Neurosurgery, Wakayama Medical University; and ${ }^{5}$ Department of Neurosurgery, Wakayama Rosai Hospital, Wakayama City, Japan
\end{abstract}

\begin{abstract}
Transvenous embolization is an effective method for treating dural arteriovenous fistulas (DAVFs) of the transversesigmoid sinus (TSS). However, in cases of complicated DAVFs, it is difficult to preserve the patency of the dural sinus. The authors describe the technical details of a new reconstructive technique using transvenous balloon-assisted Onyx embolization as another treatment option in a patient with an extensive and complex DAVF of the left TSS.

A microcatheter and compliant balloon catheter were navigated into the left internal jugular vein and placed at the distal end of the DAVF in the transverse sinus. The microcatheter was placed between the vessel wall of the TSS and the balloon. After the balloon was fully inflated, Onyx-18 was injected at the periphery of the balloon in a slow, controlled, progressive, stepwise manner; the balloon and microcatheter were simultaneously withdrawn toward the sigmoid sinus, with Onyx encompassing the entirety of the complex DAVF. The Onyx refluxed into multiple arterial feeders in a distalto-proximal step-by-step manner, ultimately resulting in an Onyx tunnel. The final angiography study revealed complete obliteration of the DAVF and patency of the TSS.

The Onyx tunnel, or reconstructive transvenous balloon-assisted Onyx embolization technique, may be an effective treatment option for large, complex DAVFs of the TSS. This technique may provide another option to facilitate the complete obliteration of the DAVF while preserving the functional sinus.

https://thejns.org/doi/abs/10.3171/2017.5.JNS17287
\end{abstract}

KEY WORDS balloon-assisted embolization; dural arteriovenous fistula; Onyx embolization; transvenous embolization; vascular disorders; surgical technique

A DURAL arteriovenous fistula (DAVF) is an arteriovenous shunt located in the dural wall of an intracranial venous structure, such as a venous sinus or expanded layer of the dura mater, with a direct pathologic connection to one or more arterial vessels. Hemorrhage or venous ischemia can occur in aggressive DAVFs with retrograde cortical venous drainage.,6 The goal of treatment is complete obliteration of the fistula, correction of the venous shunting, and reversal or occlusion of the cortical venous reflux. The availability of embolic material, such as Onyx (Covidien Neurovascular), and the navigability of new microcatheters have developed both the transarterial and transvenous endovascular technique as an initial treatment option for most cases of DAVFs. ${ }^{7,9,10}$ However, in certain cases, treatment strategies remain difficult.

Most DAVFs involve the transverse-sigmoid sinus (TSS). ${ }^{13}$ Transvenous endovascular treatment is reported to be more effective than transarterial endovascular treatment and is often used as the treatment of choice for DAVFs of the TSS. ${ }^{17}$ The transvenous approach is also a pathophysiologically appropriate treatment option for the complete embolization of DAVFs, as it directly occludes the pathologic

ABBREVIATIONS DAVF = dural arteriovenous fistula; DSA = digital subtraction angiography; TSS = transverse-sigmoid sinus; $\mathrm{VA}=$ vertebral artery. SUBMITTED February 3, 2017. ACCEPTED May 30, 2017.

* Drs. Kerolus and Chung share first authorship. 

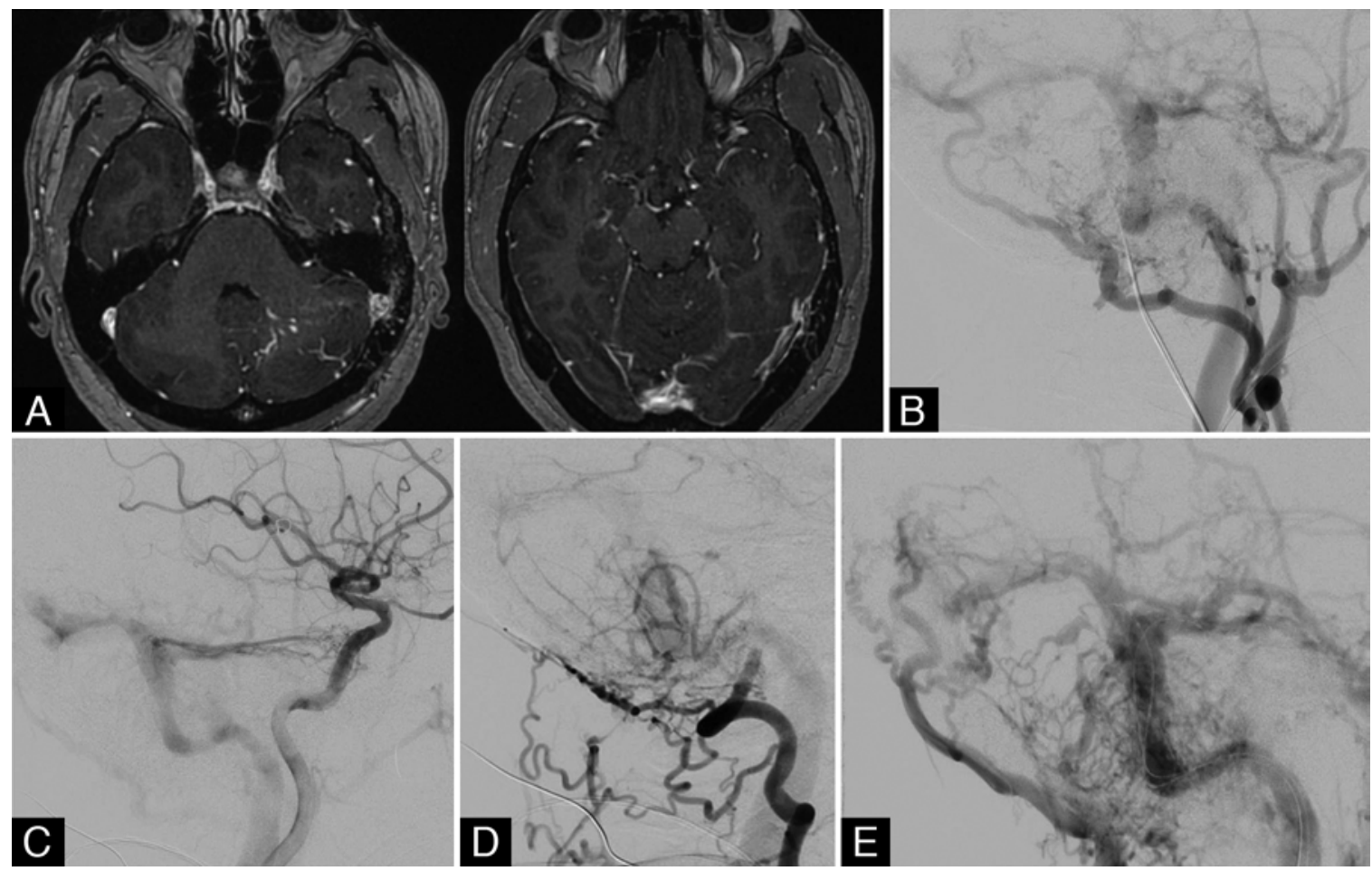

FIG. 1. A: Axial MR images demonstrating a DAVF of the left TSS, with multiple transosseous feeders and cerebellar cortical venous reflux. B: Left external carotid artery angiogram revealing multiple arterial feeders, including the middle meningeal artery, recurrent meningeal artery, scalp branches of the superficial temporal artery, and multiple transosseous branches of the occipital artery. C: Left internal carotid artery angiogram revealing arterial feeders from tentorial branches of the meningohypophyseal trunk. D: The posterior meningeal artery from the left VA was involved in the fistula as a feeder. Additionally, the left VA angiogram revealed a steal phenomenon, a cutoff sign of the left VA, meaning that the fistula was stealing blood flow from the distal VA. E: Venous phase revealing anterograde drainage to the left TSS and retrograde venous reflux that involved superficial and deep venous structures, including the vein of Labbé, superior petrosal sinus, deep medullary veins, basal vein of Rosenthal, and some cortical veins.

sinus and hence prevents cortical venous drainage and venous reflex. However, it is difficult to preserve the patency of the dural sinus in complicated DAVFs, due to its deconstructive nature and the difficulty in clearly identifying the fistulous sinus. We introduce and describe the technical details of a new reconstructive technique, the Onyx tunnel, in which transvenous balloon-assisted Onyx embolization is used to treat an extensive DAVF of the left TSS.

\section{Illustrative Case}

A 43-year-old woman presented with a long-standing history of left pulsatile tinnitus. MRI of the patient's brain revealed a DAVF of the left TSS, with multiple transosseous arterial feeders and cerebellar cortical venous reflux (Fig. 1A). Endovascular treatment for her DAVF was considered and performed based on imaging findings revealing cortical venous reflux as well as the patient's symptoms of persistent tinnitus. Digital subtraction angiography (DSA) demonstrated the presence of multiple arterial feeders into the left TSS from both the intracranial and extracranial circulations. DSA of the left external carotid artery demonstrated arterial feeders of the middle meningeal artery, recurrent meningeal artery, posterior auricular artery, scalp branches of the superficial temporal artery, and multiple transosseous branches of the left occipital artery (Fig. 1B). Additional arterial feeders were found in the tentorial branches of the meningohypophyseal trunk from the left internal carotid artery and the posterior meningeal artery from the left vertebral artery (VA) (Fig. 1C and D). DSA of the left VA revealed a cutoff sign, or steal phenomenon, in which the fistula was stealing blood flow from the VA, preventing sufficient blood flow to the distal segment of the VA (Fig. 1D). There were additional arterial feeders from branches of the right external carotid artery. The venous phase of the DSA revealed anterograde drainage into the left TSS and retrograde venous reflux involving both the superficial and deep venous structures, the vein of Labbé, superior petrosal sinus, deep medullary veins, basal vein of Rosenthal, and several cortical veins (Fig. 1E).

\section{Treatment and Technique}

Informed consent for the procedure was obtained. The patient was placed supine after induction of general anesthesia. The right common femoral artery was punctured, and a 5-Fr diagnostic catheter was used for the angiography. The left common femoral vein was punctured, and an 8-Fr guiding catheter was advanced into the left internal jugular vein. We have used the term "proximal" to describe positions close to the guiding catheter in the inter- 

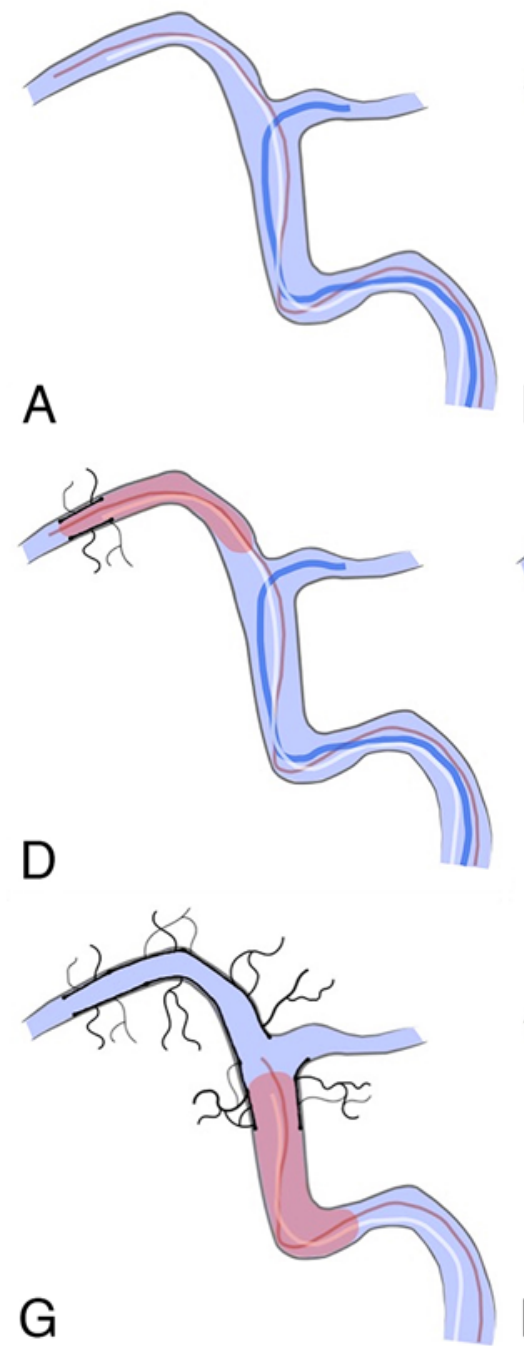
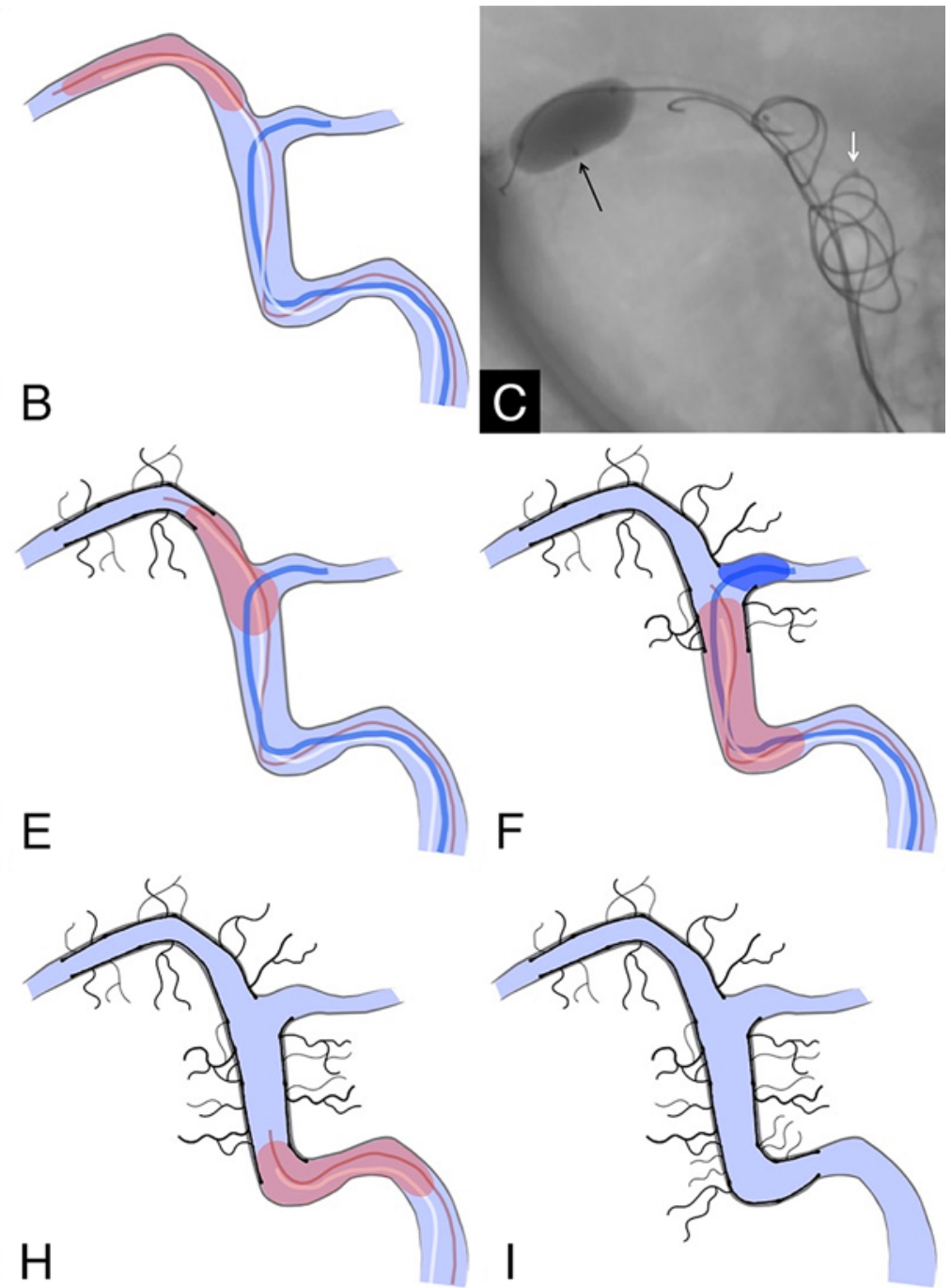

FIG. 2. Schematic description of the technique. A: A $4 \times 7$-mm HyperForm balloon (blue) was navigated into the left vein of Labbé. A second $7 \times 7-\mathrm{mm}$ HyperForm balloon ( $r e d$ ) and a microcatheter (white) were navigated to the distal end of the transverse sinus. B and C: Schematic (B) and digital subtraction angiogram $(\mathrm{C})$ demonstrating an inflated balloon in the transverse sinus (red in $\mathrm{B}$, black arrow in $\mathrm{C}$ ). The microcatheter (white in B, white arrow in C) was placed between the inflated balloon and the wall of the TSS. D: Onyx-18 (black) was then carefully injected using the microcatheter (white). E: As the inflated balloon (red) was withdrawn to the sigmoid sinus, very slow and careful injection of Onyx-18 (black) was performed in a stepwise progressive manner. F: Meanwhile, the first balloon (blue) was inflated to prevent occlusion of the vein of Labbé from Onyx infiltration. G: The first balloon was removed once the Onyx tunnel (black) was constructed around the sinus wall proximal to the vein of Labbé. $\mathrm{H}$ : The second balloon (red) was advanced in a distal-to-proximal manner until the Onyx (black) covered the entirety of the fistulas of the TSS. I: Final schematic demonstrating Onyx reflux into multiple arterial feeders and formation of an Onyx tunnel in the TSS. Copyright Joonho Chung and Mena Kerolus for all panels with the exception of panel C. Published with permission. Figure is available in color online only.

nal jugular vein, and "distal" to describe positions distant from the guiding catheter in the internal jugular vein.

The first microcatheter, an Excelsior-10 (Stryker Neurovascular), was navigated into the TSS via the left internal jugular vein. After 2 coils $(8 \times 27-\mathrm{mm}$ and $7 \times 24-$ $\mathrm{mm}$ Barricade coil system, Blockade) were deployed into the TSS, the Excelsior-10 microcatheter was placed in the left vein of Labbé to prevent its occlusion with Onyx infiltration. The second microcatheter (Marathon, Covidien Neurovascular) and a $7 \times 7-\mathrm{mm}$ HyperForm balloon (Covidien Neurovascular) were navigated to the distal end of the fistula in the transverse sinus (Fig. 2A). After the balloon was fully inflated, the Marathon microcatheter was kinked between the balloon and the wall of the TSS (Fig. 2B). Onyx-18 was then carefully injected through the Marathon microcatheter (Fig. 2C). As the inflated balloon was withdrawn toward the sigmoid sinus, injection of Onyx-18 was performed in a slow, stepwise, progressive manner at the periphery of the balloon (Fig. 2D). The microcatheter that protected the vein of Labbe was removed after the Onyx covered the proximal segment of the TSS (Fig. 2E). The balloon remained inflated and was advanced until the Onyx covered the entire TSS fistula (Fig. 2F). Onyx reflux into multiple arterial feeders in a distal-to-proximal step-by-step manner was performed, resulting in the Onyx tunnel (Fig. 2G). Final DSA of the left common carotid ar- 


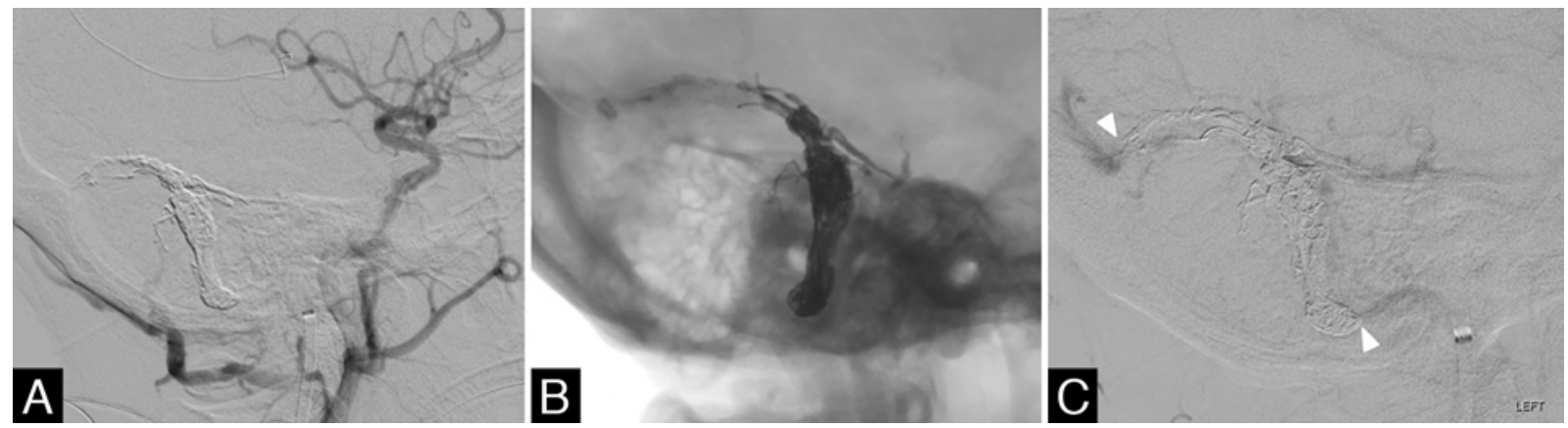

FIG. 3. Final angiograms of the left common carotid artery. A: Angiogram revealing complete obliteration of the DAVF. B: Unsubtracted image of the Onyx tunnel. C: The patency of the left TSS in the delayed venous phase. White arrowheads indicate the sinus inflow and outflow of the Onyx tunnel.

tery revealed complete obliteration of the DAVF (Fig. 3A and B) and patency of the TSS during the delayed venous phase (Fig. 3C). The bilateral femoral puncture sites were closed using Angioseal (St. Jude Medical). No antiplatelet agents were necessary before or after the procedure.

\section{Outcome and Follow-Up}

The patient recovered in the neurointensive care unit and was discharged on the 2nd postoperative day, with complete resolution of her symptoms. DSA of the left common carotid artery during her 6-month follow-up revealed complete obliteration of the DAVF (Fig. 4A and B) and patency of the left TSS (Fig. 4C).

\section{Discussion}

In this Onyx tunnel technique, we took advantage of the structural properties of an intracranial-compliant balloon and the chemical properties of a liquid embolic material. The balloon was flexible, providing navigability into the tortuous intracranial vessels while also occupying the central lumen of the TSS. This provided scaffolding for the formation of an Onyx tunnel while also providing counterpressure against the dural arteries during Onyx injection. The liquid embolic material used in this case was particularly useful, given its nonadhesive nature. When injected, the outer polymer precipitates, while the core remains liquid and does not fragment. This allows longer, slower, intermittent, controlled injections, facilitating the safe embolization of a DAVF and thus resulting in a high success rate..$^{4,5,16}$ Therefore, by using a balloon to secure the Onyx against the normal sinus wall, we were able to create a $360^{\circ}$ tunnel against the TSS wall.

A thorough understanding of the venous channel architecture is essential for successful treatment of DAVFs of the TSS. If the fistulous segment of the venous sinus is clearly identified, selective transvenous embolization of the fistulous sinus is possible without occluding the normal dural sinus. ${ }^{12}$ However, in cases of complicated DAVFs, it might be difficult to identify the entire diseased segment of the sinus to provide complete embolization. Even if the segment is clearly identified, complete embolization may not be achieved. In such cases, combined treatment options have been recommended, including transarterial endovascular treatment followed by microsurgery or ra- diosurgery; however, this combined approach may still not guarantee an easy, safe, or complete treatment. 2,7,8,12,15

Several endovascular techniques have been introduced to treat complicated DAVFs. ${ }^{14}$ Choi et al. reported the first reconstructive transvenous treatment using a stent graft for a DAVF of the transverse sinus in a patient with hypoplasia of the contralateral venous sinuses. ${ }^{3}$ Stenting provided the needed patency of the dural sinus and was successful because the fistulous segment did not involve the vein of Labbé or other large, dependent venous vasculature. However, in cases of large draining vessels involving the fistulous sinus, as in our case with the vein of Labbé, stenting may not be applicable, as occlusion of a large draining vein may preclude to a venous stroke or hemorrhage.

The transarterial approach for endovascular treatment may also be used to treat complicated DAVFs. Liquid embolic material is used to occlude arterial feeders as it allows better control and more accurate injection than the transvenous approach. Transvenous balloon-assisted transarterial injection techniques with Onyx embolization have been used with success. ${ }^{11,18}$ However, the transarterial approach does have limitations for large and complex DAVFs. Inadvertent embolization may occur and result in iatrogenic ischemia. In addition, not every arterial feeder can be selected using a microcatheter due to the increased tortuosity of the arterial feeders, the number of arterial feeders, and the small diameter of the arterial feeders. .,10 $^{5}$ Finally, not all arterial feeders may be visible on angiography. In our case, we introduce yet another technique, an Onyx tunnel, which may provide an additional treatment option to overcome complicated DAVFs of the TSS while maintaining patency of the diseased sinus and essential draining veins.

To perform the technique used in our case, there are several points to consider. First, it is difficult to achieve an end-on view or a down-barrel view to confirm the patency of the TSS because the TSS is a long and tortuous channel, limiting the view range of the angiography machine. Thus, we did not deflate the balloon while injecting the Onyx. Second, it may be beneficial to use a longer and larger balloon, such as the Copernic RC balloon (Balt), as it may facilitate embolization, increase the likelihood of a complete cure, reduce the risk when advancing the inflated balloon through the TSS, and serve as an alter- 

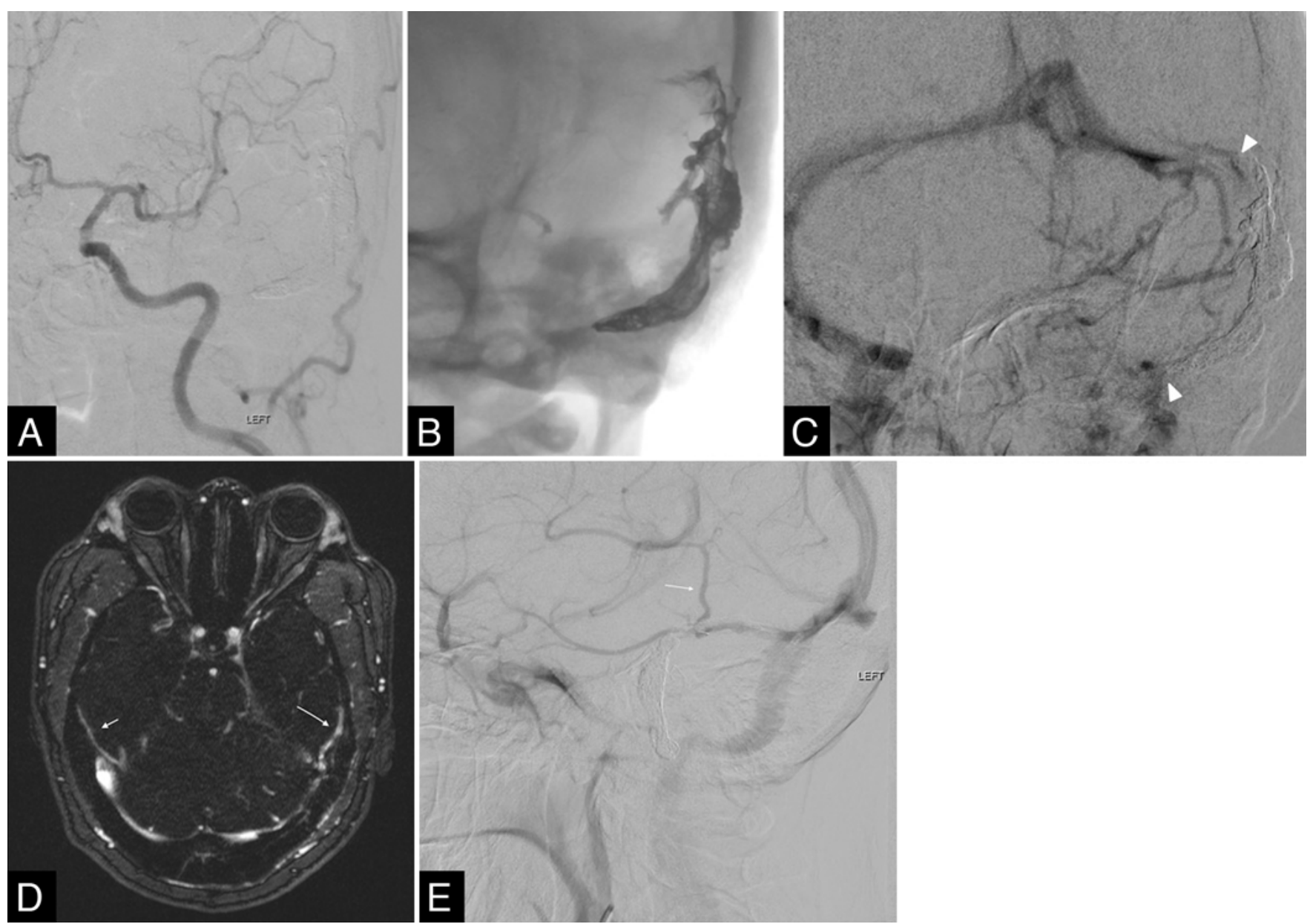

FIG. 4. Six-month follow-up angiogram studies. A: Angiogram (oblique view) of the left common carotid artery, revealing complete obliteration of the DAVF. B: Unsubtracted image of the Onyx tunnel. C: Patency of the left TSS in the delayed venous phase. White arrowheads indicate the sinus inflow and outflow of the Onyx tunnel. D: MR venogram demonstrating patency of both the left vein of Labbé (long arrow) and right vein of Labbé (short arrow). E: Digital subtraction angiogram demonstrating patency of the left vein of Labbé.

native to the initial confirmation of TSS patency. Third, the microcatheter used for the Onyx injection (e.g., the Marathon in the present case) should not be placed distal to the balloon catheter because the transverse sinus might be occluded by the Onyx. This is avoided by placing the microcatheter in the middle of the inflated balloon, and the initial Onyx injected forms a natural plug at the distal end of the balloon. Fourth, it is important to know when to withdraw the microcatheter protecting the vein of Labbé. The balloon protecting the vein of Labbé will need to be removed either when the Onyx tunnel proximal to the vein of Labbé has been sufficiently reconstructed or when the Onyx begins to fill the vein of Labbé. However, protecting the vein may not always be necessary because it may not be needed for normal venous drainage. However, it is essential to preserve functionally dependent veins or sinuses with normal venous drainage to present venous infarction or hemorrhage. Using an additional balloon and knowledge of when to remove the microcatheter may help prevent this complication. Fifth, given the large size of the venous sinus, we did not believe it was necessary to use antiplatelet medications. Aspirin or other single-agent an- tiplatelet medications could be considered to decrease the risk of thrombosis, although thrombosis did not occur in an acute or delayed fashion.

Although balloon-assisted Onyx embolization via a transarterial and transvenous route has been established, the Onyx tunnel technique offers an opportunity to preserve the sinus while providing complete embolization of the DAVF. However, this has been limited to a single case experience. Future modifications with different-sized balloons or microcatheters may be used to treat DAVFs on a case-by-case basis.

\section{Conclusions}

Reconstructive transvenous balloon-assisted Onyx embolization, the Onyx tunnel technique, may be an effective treatment option for large, complex DAVFs of the TSS. We present a case in which this technique was introduced to successfully obliterate the fistula while maintaining vessel patency of the essential draining veins. A transvenous, sinus reconstructive Onyx tunnel technique may provide another option for the complete obliteration of a DAVF. 


\section{References}

1. Awad IA, Little JR, Akarawi WP, Ahl J: Intracranial dural arteriovenous malformations: factors predisposing to an aggressive neurological course. J Neurosurg 72:839-850, 1990

2. Barnwell SL, Halbach VV, Higashida RT, Hieshima G, Wilson CB: Complex dural arteriovenous fistulas. Results of combined endovascular and neurosurgical treatment in 16 patients. J Neurosurg 71:352-358, 1989

3. Choi BJ, Lee TH, Kim CW, Choi CH: Reconstructive treatment using a stent graft for a dural arteriovenous fistula of the transverse sinus in the case of hypoplasia of the contralateral venous sinuses: technical case report. Neurosurgery 65:E994-E996, 2009

4. Clarençon F, Di Maria F, Gabrieli J, Carpentier A, Pistochi $\mathrm{S}$, Bartolini B, et al: Double-lumen balloon for Ony $\mathrm{x}^{\circledR}$ embolization via extracranial arteries in transverse sigmoid dural arteriovenous fistulas: initial experience. Acta Neurochir (Wien) 158:1917-1923, 2016

5. Cognard C, Januel AC, Silva NA Jr, Tall P: Endovascular treatment of intracranial dural arteriovenous fistulas with cortical venous drainage: new management using Onyx. AJNR Am J Neuroradiol 29:235-241, 2008

6. Endo S, Kuwayama N, Takaku A, Nishijima M: Direct packing of the isolated sinus in patients with dural arteriovenous fistulas of the transverse-sigmoid sinus. J Neurosurg 88:449-456, 1998

7. Friedman JA, Pollock BE, Nichols DA, Gorman DA, Foote RL, Stafford SL: Results of combined stereotactic radiosurgery and transarterial embolization for dural arteriovenous fistulas of the transverse and sigmoid sinuses. J Neurosurg 94:886-891, 2001

8. Goto K, Sidipratomo P, Ogata N, Inoue T, Matsuno H: Combining endovascular and neurosurgical treatments of highrisk dural arteriovenous fistulas in the lateral sinus and the confluence of the sinuses. J Neurosurg 90:289-299, 1999

9. Halbach VV, Higashida RT, Hieshima GB, Goto K, Norman $\mathrm{D}$, Newton TH: Dural fistulas involving the transverse and sigmoid sinuses: results of treatment in 28 patients. Radiology 163:443-447, 1987

10. Hu YC, Newman CB, Dashti SR, Albuquerque FC, McDougall CG: Cranial dural arteriovenous fistula: transarterial Onyx embolization experience and technical nuances. J Neurointerv Surg 3:5-13, 2011

11. Jittapiromsak P, Ikka L, Benachour N, Spelle L, Moret J: Transvenous balloon-assisted transarterial Onyx embolization of transverse-sigmoid dural arteriovenous malformation. Neuroradiology 55:345-350, 2013

12. Kirsch M, Liebig T, Kühne D, Henkes H: Endovascular man- agement of dural arteriovenous fistulas of the transverse and sigmoid sinus in 150 patients. Neuroradiology 51:477-483, 2009

13. Kiyosue H, Hori Y, Okahara M, Tanoue S, Sagara Y, Matsumoto S, et al: Treatment of intracranial dural arteriovenous fistulas: current strategies based on location and hemodynamics, and alternative techniques of transcatheter embolization. Radiographics 24:1637-1653, 2004

14. Liebig T, Henkes H, Brew S, Miloslavski E, Kirsch M, Kühne D: Reconstructive treatment of dural arteriovenous fistulas of the transverse and sigmoid sinus: transvenous angioplasty and stent deployment. Neuroradiology 47:543-551, 2005

15. Pollock BE, Nichols DA, Garrity JA, Gorman DA, Stafford SL: Stereotactic radiosurgery and particulate embolization for cavernous sinus dural arteriovenous fistulae. Neurosurgery 45:459-467, 1999

16. Shi ZS, Loh Y, Duckwiler GR, Jahan R, Viñuela F: Balloonassisted transarterial embolization of intracranial dural arteriovenous fistulas. J Neurosurg 110:921-928, 2009

17. Webb S, Hopkins LN: Intracranial dural arteriovenous fistulas: a treatment paradigm in flux. World Neurosurg 80:4749, 2013

18. Zhang Y, Li Q, Huang QH: Embolization of a superior sagittal sinus dural arteriovenous fistula under intrasinus balloon protection: A case report. Interv Neuroradiol 21:94-100, 2015

\section{Disclosures}

Dr. Lopes reports being a consultant for Medtronic, Stryker, and Siemens.

\section{Author Contributions}

Conception and design: Lopes, Kerolus, Chung, Munich, Matsuda. Acquisition of data: Lopes, Kerolus, Chung, Munich, Matsuda. Analysis and interpretation of data: all authors. Drafting the article: Kerolus, Chung, Munich, Matsuda, Okada. Critically revising the article: all authors. Reviewed submitted version of manuscript: Lopes, Kerolus, Chung, Munich, Matsuda. Administrative/techni$\mathrm{cal} /$ material support: Kerolus, Munich, Matsuda, Okada. Study supervision: Lopes.

\section{Correspondence}

Demetrius Lopes, Department of Neurosurgery, Rush University Medical Center, 1725 W Harrison St., Ste. 855, Chicago, IL 60612. email: demetrius_lopes@rush.edu. 